\title{
THERAPEUTIC POTENTIAL OF TRAMADOL AND DEXTROMETHORPHAN ON VINCRISTINE INDUCED PERIPHERAL NEUROPATHY IN RATS
}

\author{
CRISTINA ELENA ZBÂRCEA ${ }^{1 *}$, CORNEL CHIRIȚĂ ${ }^{1 \#}$, EMIL ȘTEFĂNESCU ${ }^{1 \#}$, OANA \\ CRISTINA ȘEREMET ${ }^{1 * *}$, BRUNO ȘTEFAN VELESCU ${ }^{1 *}$, CRISTINA DANIELA MARINECI ${ }^{1 \#}$, \\ OVIDIU MUŞAT ${ }^{2 \#}$, CARMEN GIUGLEA ${ }^{3 \#}$, SIMONA NEGREŞ ${ }^{1 \#}$
}

${ }^{I}$ Pharmacology and Clinical Pharmacy Department, Faculty of Pharmacy, University of Medicine and Pharmacy "Carol Davila”, 6 Traian Vuia Street, 020956, Bucharest, Romania

${ }^{2}$ Central Military Emergency Hospital, 139 Calea Plevnei Street, 010825 Bucharest, Romania

3 "Sf. Ioan" Clinical Emergency Hospital, 13 Vitan Bârzești Road, 042122, Bucharest, Romania

*corresponding author: oana.seremet@yahoo.com

\#Authors with equal contribution

Manuscript received: September 2018

\begin{abstract}
Vincristine is prescribed in many neoplastic diseases such as acute lymphoblastic leukaemia, Hodgkin's disease, nonHodgkin's lymphomas, breast cancer, small cell lung cancer, cervical cancer, multiple myeloma. The neurotoxicity of vincristine is severe, being a dose limiting factor that depends on the cumulative dose and the frequency of administration. Data from clinical and preclinical studies show therapeutic potential for atypical opioid analgesics in neuropathic pain. In this research we investigated the analgesic potential for tramadol and dextromethorphan in animal model of vincristine-induced peripheral neuropathy. The peripheral neuropathy was induced in male Wistar rats by the daily intraperitoneal (i.p.) administration of vincristine sulphate $0.1 \mathrm{mg} / \mathrm{kg} /$ day, 5 days followed by 2 days break and then another 5 days of vincristine. Analgesics were given daily seven days after the vincristine treatment in the following doses: tramadol $5 \mathrm{mg} / \mathrm{kg} \mathrm{bw}$, dextromethorphan $20 \mathrm{mg} / \mathrm{kg}$ bw. We tracked the evolution of allodynia and mechanical hyperalgesia under analgesic treatment. Following the processing of experimental data, the first dose of tramadol significantly increased maximum response time, both in the assessment of allodynia and hyperalgesia. A single dose of dextromethorphan produced a significant inhibition of allodynia induced by vincristine (increases the time to response for paw retraction with $32.65 \%$, p $<$ 0.001 , vs vincristine group). Tramadol lead to the maximum anti-allodynic effect, immediately after the discontinuation of vincristine $(141.73 \%, \mathrm{p}<0.001)$. Tramadol showed therapeutic potential in combating neuropathic pain induced by the administration of Vinca alkaloids.
\end{abstract}

\section{Rezumat}

Vincristina este prescrisă în numeroase bolile neoplazice precum: leucemie limfoblastică acută, boală Hodgkin, limfoame non-Hodgkin, cancer mamar, cancer pulmonar cu celule mici, cancer de col uterin, mielom multiplu. Neurotoxicitatea vincristinei este severă fiind un factor care limitează doza și este influențată de doza cumulativă și de frecvența dintre administrări. Date din studiile clinice și preclinice arată potențialul terapeutic al analgezicelor opioide atipice în durerea neuropată. În aceast studiu am investigat potențialul analgezic pentru tramadol și dextrometorfan în modelul experimental de neuropatie periferică indusă de vincristină. Neuropatia periferică a fost indusă la şobolani masculi Wistar prin administrare zilnică intraperitoneală (i.p.) a sulfatului de vincristină $0,1 \mathrm{mg} / \mathrm{kg} / \mathrm{zi}, 5$ zile, o pauză de 2 zile și apoi încă 5 zile de vincristină. Analgezicele au fost administrate zilnic la șapte zile după tratamentul cu vincristină în următoarele doze: tramadol $5 \mathrm{mg} / \mathrm{kg}$ corp, dextrometorfan $20 \mathrm{mg} / \mathrm{kg}$ corp. Am urmărit evoluția alodiniei și hiperalgeziei mecanice în urma tratamentului cu analgezice. În urma prelucrării datelor experimentale, prima doză de tramadol a crescut semnificativ timpul de răspuns, atât în evaluarea alodiniei cât și a hiperalgeziei. Doar după administrarea unei doze unice, dextrometorfanul a produs o inhibare semnificativă a alodiniei induse de vincristină (crește timpul de reacție al labei cu 32,65\%, p < 0,001, față de lotul vincristină). Tramadolul produce efect maxim anti-alodinic, imediat după întreruperea administrării vincristinei $(141,73 \%, p$ $<0,001)$. Tramadolul prezintă potențial terapeutic în combaterea durerii neuropate indusă de utilizarea alcaloizilor din Vinca minor.

Keywords: tramadol, dextromethorphan, vincristine, peripheral neuropathy

\section{Introduction}

In the context of cancer, neuropathic pain may be a late effect of treatment with Vinca alkaloids, taxanes, platinum-derived compounds, radiotherapy, antibodies, or surgery [1]. The peripheral neuropathy is the adverse reaction whose toxicity limits the dose for many chemotherapeutic agents [2- 
6]. The mechanisms claiming chemotherapy-induced peripheral neuropathy are diverse and depend on the drug. Vincristine- and paclitaxel-induced painful peripheral neuropathies are accompanied by a reduction of intraepidermal nerve fiber (IENF) density corresponding to the onset of mechanical hypersensitivity $[7,8]$.

The available options for the management of neuropathic pain imply multiple lines of therapy: opioid analgesics, antidepressants, anticonvulsants, N-methyl-D-aspartate (NMDA) receptor antagonists, gamma-aminobutyric acid (GABA) B receptor agonists and topical capsaicin [9].

New chemical structures are being studied for potential analgesic effect in neuropathy $[10,11]$. The animal models are an important source of in vivo information and can be used to evaluate the efficacy and safety of drugs and may provide significant information for clinical use.

In this research we chose to investigate the efficacy of two opioid analgesics with complex mechanism: dextromethorphan (an opioid agonist and antagonist of the NMDA receptor) and tramadol (a weak opioid agonist and a serotonin-norepinephrine reuptake inhibitor (SNRI)) in vincristine-induced peripheral neuropathy.

Tramadol is suggested by most guidelines for second-line treatment of neuropathic pain [12] and is also a therapeutic option for the control of neuropathic pain in patients with cancer [13]. In our previous research administration of tramadol (5 $\mathrm{mg} / \mathrm{kg} \mathrm{bw}$ ) reduced statistically significant also the allodynia and hyperalgesia induced by paclitaxel [14]. We also showed that tramadol and dextromethorphan reduce the vincristine induced thermal hyperalgesia [15].

\section{Materials and Methods}

\section{Animals}

We used adult male Wistar rats $(182.3 \pm 29.11 \mathrm{~g}, \mathrm{~N}$ $=48$ ). They were supplied by the rodent farm of the University of Medicine and Pharmacy "Carol Davila". The tests were performed between 8:00 a.m. and 2:00 p.m. Before testing, all animals were allowed to get used to the testing environment. During this preclinical study the bioethics norms imposed by the legislation (2010/63/UE/22.09.2010) were followed. The experimental proceedings were approved by the Faculty of Pharmacy, "Carol Davila" Medicine and Pharmacy University, Bucharest, Romania, Ethics Committee.

Rat model of vincristine-induced peripheral neuropathy We used the vincristine-evoked peripheral neuropathic model. After baseline responses to mechanical stimuli of the both hind paws, rats received intraperitoneal (i.p.) injections of vincristine sulphate $(0.1 \mathrm{mg} / \mathrm{kg} /$ day; $\mathrm{N}=36)$ or saline $(1 \mathrm{~mL} / 100 \mathrm{~g} ; \mathrm{N}=12)$ daily for 2 weeks. The vincristine was administered daily in, 5 days followed by 2 days break and then another 5 days of vincristine, as described by Weng et al. [16]. With the support of the Dynamic Plantar Aesthesiometer (Ugo Basile-Comerio, Italy) we determined the tactile hypersensitivity (allodynia and hyperalgesia).

Mechanical sensitivity

The tactile sensitivity was appraised by the instrumentality of Dynamic Plantar Aesthesiometer (Ugo Basile, Italy). The manner of work used in this experiment is what we have extensively outlined in the previously published article [14]. We measured the response following the application of two mechanical stimuli $2 \mathrm{~g} / \mathrm{s}$ (tactile allodynia) and $4 \mathrm{~g} / \mathrm{s}$ (mechanical hyperalgesia). For each mechanical stimulus, three applications were made on each posterior paw at each time point, spaced with 3 - 5 mines one from the other. The individual data are presented as the average of the six records. The force and the time needed to get a withdrawal responses are expressed, respectively, in grams and seconds.

Protocol

Assessment of tactile allodynia and mechanical hyperalgesia was performed right before (predrug), after ten doses of vincristine (day 12) and after one and seven doses of analgesics (day 15, respectively (day 21).

The four animal groups received the following: Group C: Control group $(\mathrm{N}=12)$, normal saline 0.1 $\mathrm{mL} / 100 \mathrm{~g}$ bw, i.p. on $1-5$ and $8-12$ days and distilled water, $1 \mathrm{~mL} / 100 \mathrm{~g}$ bw, p.o. on $15-21$ days; Group V: Vincristine group $(\mathrm{N}=12)$, vincristine $0.1 \mathrm{mg} / \mathrm{kg}$ bw, i.p. on $1-5$ and $8-12$ days and distilled water, $1 \mathrm{~mL} / 100 \mathrm{~g}$ bw p.o. on 15 - 21 days; Group VDMF: Vincristine and Dextromethorphan group $(\mathrm{N}=12)$, vincristine 0.1 $\mathrm{mg} / \mathrm{kg}$ bw, i.p. on $1-5$ and $8-12$ days and dextromethorphan $20 \mathrm{mg} / \mathrm{kg}$ bw, p.o. on $15-21$ days; Group VT: Vincristine and Tramadol group $(\mathrm{N}=12)$, vincristine $0.1 \mathrm{mg} / \mathrm{kg}$ bw, i.p. on $1-5$ and 8 - 12 days and tramadol $5 \mathrm{mg} / \mathrm{kg}$ bw, p.o. on 15 21 days;

\section{Statistical analysis}

Data are presented as mean \pm SEM of 12 animals per group. Results were processed using GraphPad Prism 5 software (San Diego, California, SUA, www.graphpad.com). We established distribution normality in the groups using the D'Agostino \& Pearson test. To compare the groups we used the ANOVA followed by Dunnett's (when compared to the baseline response) or Bonferroni's test (when compared to control group or vincristine group). 
FARMACIA, 2018, Vol. 66, 6

\section{Results and Discussion}

The mechanical response was assessed by two parameters: time to response (s) and applied force (g). Vincristine treatment established neuropathic states characterized by hypersensitivities to mechanical stimulation (allodynia $-2 \mathrm{~g} / \mathrm{s}$ and hyperalgesia $-4 \mathrm{~g} / \mathrm{s}$ ). The experimental results revealed that the tactile sensitivity of the animals treated with vincristine returned to baseline on day 22 (after 9 days since the 5 days followed by 2 days break and then another 5 days of vincristine).
Treatment with vincristine significantly reduced the withdrawal thresholds to mechanical allodynia (reduces the time to response) on day 12 (13.01 \pm $1.19 \mathrm{~s})$ compared to the control mechanical thresholds $(22.32 \pm 2.22 \mathrm{~s})(\mathrm{p}<0.01$; Table I; Figure 1).

Oral administration of tramadol $(5 \mathrm{mg} / \mathrm{kg} \mathrm{bw})$ and dextromethorphan $(20 \mathrm{mg} / \mathrm{kg}$ bw) produced a significant inhibition of mechanical allodynia induced by vincristine (increases the time to response for paw retraction) on day 15 compared to vincristine group $(\mathrm{p}<0.001$; Figure 2$)$.

Table I

Modifications in time to response and applied force during allodynia (2g/s) following vincristine and each of the drug treatment: dextromethorphan $(20 \mathrm{mg} / \mathrm{kg} \mathrm{bw})$ or tramadol $(5 \mathrm{mg} / \mathrm{kg} \mathrm{bw})$

\begin{tabular}{|c|c|c|c|c|c|c|c|c|c|}
\hline \multirow{3}{*}{ ํㅡㄹ } & & \multicolumn{8}{|c|}{ Behavioural tactile allodynia in response to stimulus $2 \mathrm{~g} / \mathrm{s}$} \\
\hline & & \multicolumn{2}{|c|}{ Basal } & \multicolumn{2}{|c|}{ Day 12} & \multicolumn{2}{|c|}{ Day 15} & \multicolumn{2}{|c|}{ Day 21} \\
\hline & & $\begin{array}{l}\text { Time } \\
\text { (s) }\end{array}$ & $\begin{array}{c}\text { Force } \\
\text { (g) }\end{array}$ & $\begin{array}{c}\text { Time } \\
\text { (s) }\end{array}$ & $\begin{array}{c}\text { Force } \\
\text { (g) }\end{array}$ & Time (s) & $\begin{array}{c}\text { Force } \\
\text { (g) }\end{array}$ & Time (s) & $\begin{array}{c}\text { Force } \\
\text { (g) }\end{array}$ \\
\hline \multirow{3}{*}{ 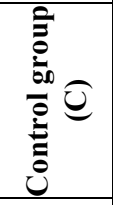 } & $\begin{array}{l}\text { Mean/group } \pm \\
\text { SEM }\end{array}$ & $\begin{array}{c}19.07 \pm \\
1.77\end{array}$ & $\begin{array}{c}19.32 \pm \\
0.28\end{array}$ & $\begin{array}{c}16.17 \pm \\
1.57\end{array}$ & $\begin{array}{c}18.91 \pm \\
0.38\end{array}$ & $\begin{array}{c}22.32 \pm \\
2.22\end{array}$ & $\begin{array}{c}19.63 \pm \\
0.14\end{array}$ & $\begin{array}{c}18.84 \pm \\
1.93\end{array}$ & $\begin{array}{c}19.29 \pm \\
0.22\end{array}$ \\
\hline & \multicolumn{3}{|c|}{ Procentual change vs baseline } & -15.21 & -2.12 & 17.07 & 1.6 & -1.21 & -0.16 \\
\hline & \multicolumn{3}{|c|}{$\begin{array}{l}\text { ANOVA test followed by Dunnett's post-test } \\
\qquad v s \text { baseline }(\mathrm{p})\end{array}$} & ns & ns & ns & ns & ns & ns \\
\hline \multirow{5}{*}{ 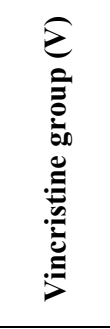 } & $\begin{array}{l}\text { Mean/group } \pm \\
\text { SEM }\end{array}$ & $\begin{array}{l}20.58 \pm \\
2.56\end{array}$ & $\begin{array}{l}19.53 \pm \\
0.19\end{array}$ & $\begin{array}{l}13.57 \pm \\
1.19\end{array}$ & $\begin{array}{c}18.12 \pm \\
0.71\end{array}$ & $\begin{array}{l}13.01 \pm \\
1.19\end{array}$ & $\begin{array}{c}18.43 \pm \\
0.49\end{array}$ & $\begin{array}{l}17.54 \pm \\
1.00\end{array}$ & $\begin{array}{c}19.44 \pm \\
0.13\end{array}$ \\
\hline & \multicolumn{3}{|c|}{ Procentual change vs baseline } & -34.06 & -7.22 & -36.78 & -5.63 & -14.77 & -0.46 \\
\hline & \multicolumn{3}{|c|}{$\begin{array}{l}\text { ANOVA test followed by Dunnett's post-test } \\
\qquad v s \text { baseline (p) }\end{array}$} & $*$ & ns & $* *$ & ns & ns & ns \\
\hline & \multicolumn{3}{|c|}{ Procentual change vs control group } & -18.86 & -5.10 & -53.83 & -7.24 & -13.57 & -0.31 \\
\hline & \multicolumn{3}{|c|}{$\begin{array}{l}\text { ANOVA test followed by Bonferroni's post- } \\
\text { test } v s \text { control group (p) }\end{array}$} & ns & ns & $* * *$ & ns & ns & ns \\
\hline \multirow{7}{*}{ 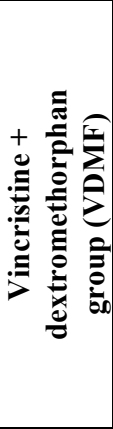 } & $\begin{array}{l}\text { Mean/group } \pm \\
\text { SEM }\end{array}$ & $\begin{array}{l}19.36 \pm \\
2.56\end{array}$ & $\begin{array}{c}19.16 \pm \\
0.18\end{array}$ & $\begin{array}{c}12.17 \pm \\
1.83\end{array}$ & $\begin{array}{c}16.06 \pm \\
1.19\end{array}$ & $\begin{array}{c}18.56 \pm \\
1.47\end{array}$ & $\begin{array}{c}19.54 \pm \\
0.26\end{array}$ & $\begin{array}{c}18.98 \pm \\
1.80\end{array}$ & $\begin{array}{c}19.18 \pm \\
0.31 \\
\end{array}$ \\
\hline & \multicolumn{3}{|c|}{ Procentual change vs baseline } & -37.14 & -16.18 & -4.31 & 1.98 & -1.96 & $\mathbf{0 , 1 0}$ \\
\hline & \multicolumn{3}{|c|}{$\begin{array}{l}\text { ANOVA test followed by Dunnett's post-test } \\
\qquad v s \text { baseline (p) }\end{array}$} & $*$ & ns & ns & ns & ns & ns \\
\hline & \multicolumn{3}{|c|}{ Procentual change vs control group } & -21.93 & -14.66 & -21.17 & 0.38 & -0.76 & 0.26 \\
\hline & \multicolumn{3}{|c|}{$\begin{array}{l}\text { ANOVA test followed by Bonferroni's post- } \\
\text { test } v s \text { control group }(\mathrm{p})\end{array}$} & ns & ns & ns & ns & ns & ns \\
\hline & \multicolumn{3}{|c|}{ Procentual change vs vincristine group } & -3.08 & -8.96 & 32.65 & 7.62 & 12.81 & 0.57 \\
\hline & \multicolumn{3}{|c|}{$\begin{array}{l}\text { ANOVA test followed by Bonferroni's post- } \\
\text { test } v s \text { control group (p) }\end{array}$} & ns & ns & $* * *$ & ns & ns & ns \\
\hline \multirow{7}{*}{ 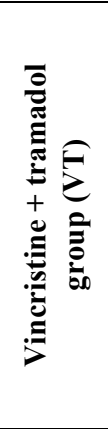 } & $\begin{array}{l}\text { Mean/group } \pm \\
\text { SEM }\end{array}$ & $\begin{array}{c}16.62 \pm \\
1.82 \\
\end{array}$ & $\begin{array}{r}19.14 \\
\pm 0.33 \\
\end{array}$ & $\begin{array}{c}12.31 \pm \\
1.99 \\
\end{array}$ & $\begin{array}{c}15.94 \pm \\
1.12 \\
\end{array}$ & $\begin{array}{c}40.21 \pm \\
6.23\end{array}$ & $\begin{array}{c}18.98 \pm \\
0.46\end{array}$ & $\begin{array}{c}37.57 \pm \\
4.98 \\
\end{array}$ & $\begin{array}{c}19.64 \pm \\
0.19 \\
\end{array}$ \\
\hline & \multicolumn{3}{|c|}{ Procentual change vs baseline } & $-37,26$ & -16.72 & 104.94 & -0.84 & 91.49 & 2.61 \\
\hline & \multicolumn{3}{|c|}{$\begin{array}{l}\text { ANOVA test followed by Dunnett's post-test } \\
\qquad v s \text { baseline (p) }\end{array}$} & $* *$ & $* *$ & $* *$ & ns & $* * *$ & ns \\
\hline & \multicolumn{3}{|c|}{ Procentual change vs control group } & -22.05 & -14.60 & 87.90 & -2.44 & 92.69 & 2.77 \\
\hline & \multicolumn{3}{|c|}{$\begin{array}{l}\text { ANOVA test followed by Bonferroni's post- } \\
\text { test } v s \text { control group (p) }\end{array}$} & ns & ns & $* * *$ & ns & $* * *$ & ns \\
\hline & \multicolumn{3}{|c|}{ Procentual change vs vincristine group } & -3.20 & -9.50 & 141.73 & 4.8 & 106.26 & 3.07 \\
\hline & \multicolumn{3}{|c|}{$\begin{array}{l}\text { ANOVA test followed by Bonferroni's post- } \\
\text { test } v s \text { paclitaxel group (p) }\end{array}$} & ns & ns & $* * *$ & ns & $* * *$ & ns \\
\hline
\end{tabular}




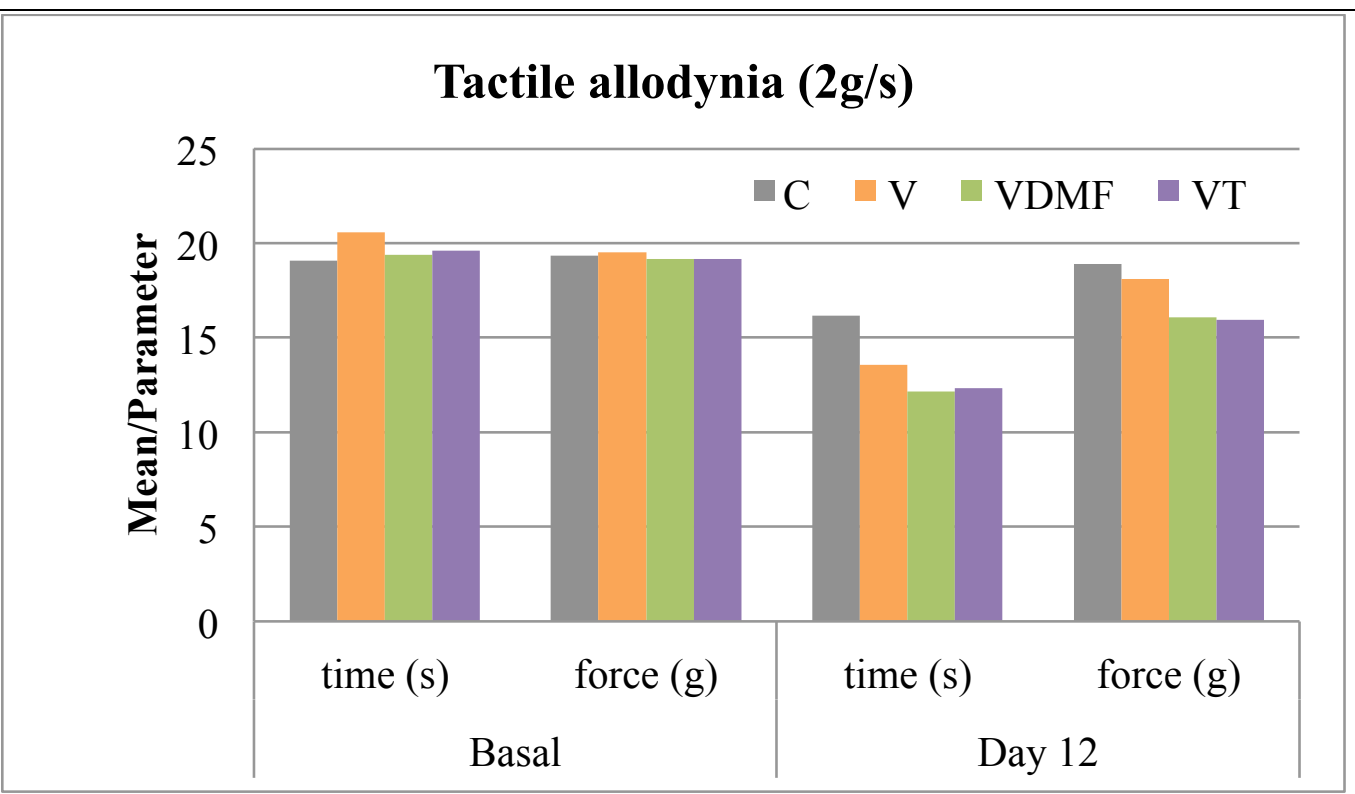

Figure 1.

Behavioural tactile allodynia induced with vincristine, shown as the mean of the time to response and applied force, to mechanical stimulation $2 \mathrm{~g} / \mathrm{s}$

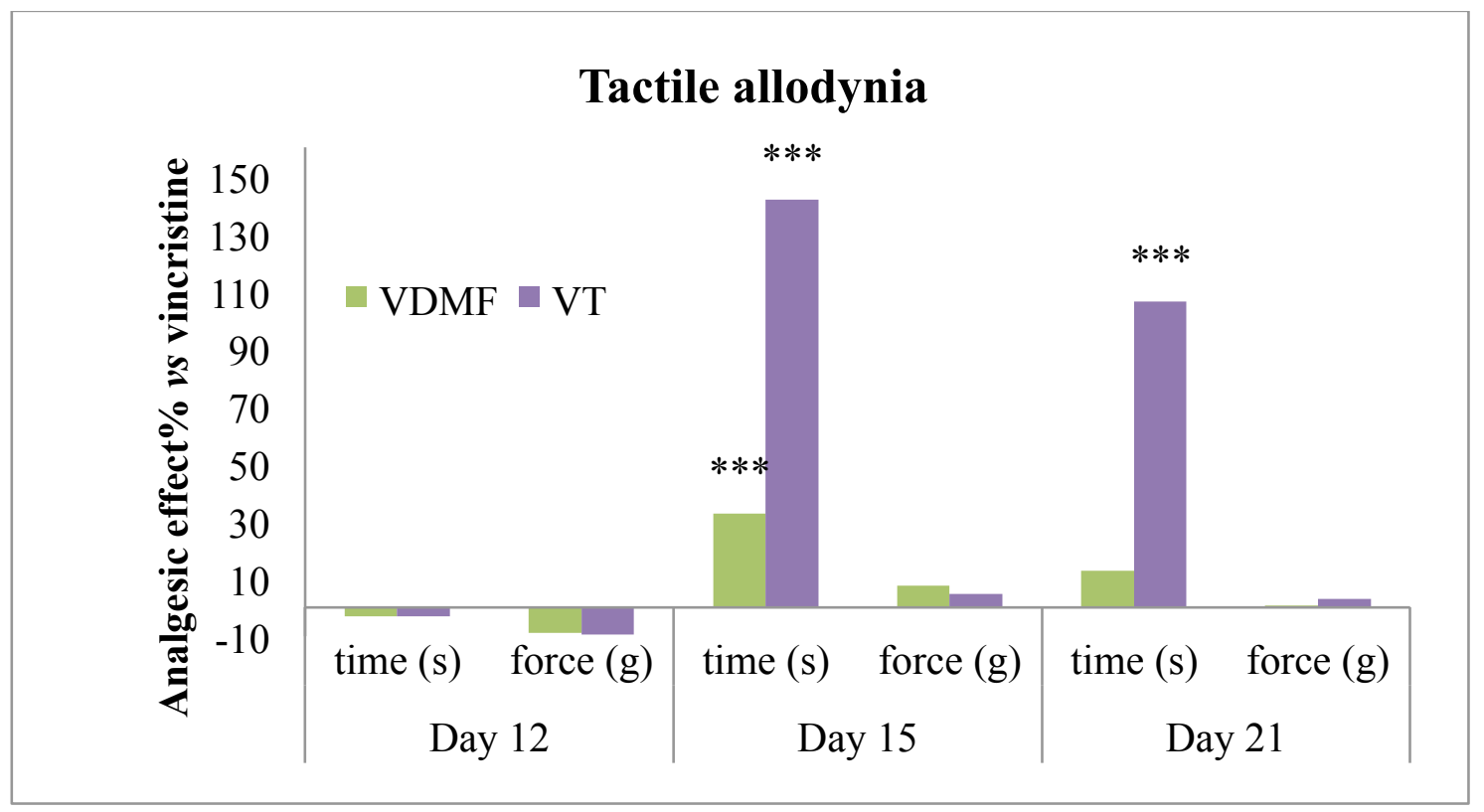

Figure 2.

Analgesic effect (\%) of dextromethorphan $(20 \mathrm{mg} / \mathrm{kg} \mathrm{bw})$ and tramadol $(5 \mathrm{mg} / \mathrm{kg}$ bw) in the tactile allodynia induced with vincristine. Group comparisons were performed using the One-Way analysis of variance ANOVA followed by Bonferroni's test in comparison to vincristine group $* * * p<0.001$

Vincristine produced a significant reduction in response withdrawal thresholds to mechanical stimulus of $4 \mathrm{~g} / \mathrm{s}$ (mechanical hyperalgesia), on days 12 and 15 after first drug administration compared to the baseline and control group (Table II, Figure 3). Vincristine-induced mechanical hyperalgesia in rats was decreased by administration of tramadol $(5 \mathrm{mg} /$ $\mathrm{kg} \mathrm{bw})$. The two parameters, the time to response and the force applied for paw retraction were changed significant on day $15(8.70 \pm 1.17 \mathrm{~s} ; \mathrm{p}<0.01 ; 33.41 \pm$ $3.35 \mathrm{~g} \mathrm{p}<0.001)$ compared to vincristine group (Table II; Figure 4).

The treatment with dextromethorphan $(5 \mathrm{mg} / \mathrm{kg}$ bw) did not reduce the mechanical hyperalgesia caused by vincristine (Figure 4 ). 
Table II

Modifications in time to response and applied force during hyperalgesia $(4 \mathrm{~g} / \mathrm{s})$ following vincristine and each of the drug treatment: dextromethorphan $(20 \mathrm{mg} / \mathrm{kg} \mathrm{bw})$ or tramadol $(5 \mathrm{mg} / \mathrm{kg} \mathrm{bw})$

\begin{tabular}{|c|c|c|c|c|c|c|c|c|c|}
\hline \multirow[b]{3}{*}{$\stackrel{\Xi}{0}$} & & \multicolumn{8}{|c|}{ Behavioural tactile hyperalgesia in response to stimulus $4 \mathrm{~g} / \mathrm{s}$} \\
\hline & & \multicolumn{2}{|l|}{ Basal } & \multicolumn{2}{|l|}{ Day 12} & \multicolumn{2}{|l|}{ Day 15} & \multicolumn{2}{|l|}{ Day 21} \\
\hline & & $\begin{array}{l}\text { Time } \\
\text { (s) }\end{array}$ & $\begin{array}{l}\text { Force } \\
(\mathrm{g})\end{array}$ & $\begin{array}{l}\text { Time } \\
\text { (s) }\end{array}$ & $\begin{array}{l}\text { Force } \\
\text { (g) }\end{array}$ & $\begin{array}{l}\text { Time } \\
\text { (s) }\end{array}$ & $\begin{array}{l}\text { Force } \\
\text { (g) }\end{array}$ & $\begin{array}{l}\text { Time } \\
\text { (s) }\end{array}$ & $\begin{array}{l}\text { Force } \\
\text { (g) }\end{array}$ \\
\hline \multirow{3}{*}{ 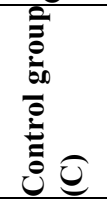 } & $\begin{array}{ll}\text { Mean/group } \quad \pm \\
\text { SEM }\end{array}$ & \begin{tabular}{ll|}
8.05 & \pm \\
0.39 &
\end{tabular} & $\begin{array}{l}32.01 \\
1.41\end{array}$ & $\begin{array}{ll}8.51 & \pm \\
0.39 & \end{array}$ & $\begin{array}{ll}32.36 \pm \\
1.04\end{array}$ & $\begin{array}{ll}8.06 & \pm \\
0.18 & \end{array}$ & $\begin{array}{l}31.92 \pm \\
0.63\end{array}$ & $\begin{array}{ll}8.37 & \pm \\
0.38 & \end{array}$ & $\begin{array}{ll}33.51 \pm \\
0.71\end{array}$ \\
\hline & \multicolumn{3}{|c|}{ Procentual change vs baseline } & 5.80 & 1.09 & 0.11 & -0.28 & 3.99 & 4.69 \\
\hline & \multicolumn{3}{|c|}{$\begin{array}{l}\text { ANOVA test followed by Dunnett's post-test } \\
v s \text { baseline }(\mathrm{p})\end{array}$} & ns & ns & ns & ns & ns & ns \\
\hline \multirow{5}{*}{ 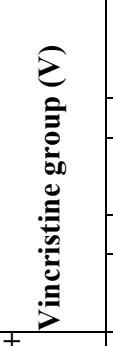 } & $\begin{array}{l}\text { Mean/group } \\
\text { SEM }\end{array}$ & $\begin{array}{ll}8.34 & \pm \\
0.47 & \end{array}$ & $\begin{array}{l}32.32 \\
1.33\end{array}$ & $\begin{array}{l}6.49 \pm \\
0.72\end{array}$ & $\begin{array}{l}25.39 \pm \\
2.51\end{array}$ & $\begin{array}{l}6.89 \pm \\
0.34\end{array}$ & $\begin{array}{l}27.16 \pm \\
1.20\end{array}$ & $\begin{array}{l}8.54 \pm \\
0.23\end{array}$ & $\begin{array}{l}34.12 \pm \\
0.87\end{array}$ \\
\hline & \multicolumn{3}{|c|}{ Procentual change vs baseline } & -22.19 & -21.25 & -17.36 & -15.76 & 2.35 & 5.83 \\
\hline & \multicolumn{3}{|c|}{$\begin{array}{l}\text { ANOVA test followed by Dunnett's post-test } \\
\text { vs baseline (p) }\end{array}$} & $*$ & $*$ & $*$ & $*$ & ns & ns \\
\hline & \multicolumn{3}{|c|}{ Procentual change vs control group } & -28.00 & -22.34 & -17.47 & -15.48 & -1.64 & 1.15 \\
\hline & \multicolumn{3}{|c|}{$\begin{array}{l}\text { ANOVA test followed by Bonferroni's post- } \\
\text { test } v s \text { control group }(\mathrm{p})\end{array}$} & $* *$ & $* * *$ & $*$ & * & ns & ns \\
\hline \multirow{7}{*}{ 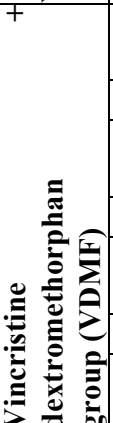 } & $\begin{array}{l}\text { Mean/group } \quad \pm \\
\text { SEM }\end{array}$ & \begin{tabular}{ll|}
8.50 & \pm \\
0.42 &
\end{tabular} & $\begin{array}{ll}32.19 \quad \pm \\
0.98 & \end{array}$ & $\begin{array}{ll}6.80 & \pm \\
0.54 & \end{array}$ & $\begin{array}{ll}26.28 \pm \\
1.77\end{array}$ & $\begin{array}{ll}6.87 & \pm \\
0.33 & \end{array}$ & $\begin{array}{l}27.36 \pm \\
1.25\end{array}$ & $\begin{array}{l}8.03 \pm \\
0.39\end{array}$ & $\begin{array}{l}31.15 \pm \\
1.32\end{array}$ \\
\hline & \multicolumn{3}{|c|}{ Procentual change vs baseline } & -20.01 & -18.36 & -19.20 & -15.00 & -5.47 & -3.23 \\
\hline & \multicolumn{3}{|c|}{$\begin{array}{l}\text { ANOVA test followed by Dunnett's post-test } \\
v s \text { baseline }(\mathrm{p})\end{array}$} & $*$ & $*$ & $*$ & $*$ & ns & ns \\
\hline & \multicolumn{3}{|c|}{ Procentual change vs control group } & -25.81 & -19.45 & -19.31 & -14.72 & -9.46 & -7.94 \\
\hline & \multicolumn{3}{|c|}{$\begin{array}{l}\text { ANOVA test followed by Bonferroni's post- } \\
\text { test } v s \text { control group }(\mathrm{p})\end{array}$} & $* *$ & $* * *$ & * & $*$ & ns & ns \\
\hline & \multicolumn{3}{|c|}{ Procentual change vs vincristine group } & 2.18 & 2.89 & -1.84 & 0.75 & -7.82 & -9.06 \\
\hline & \multicolumn{3}{|c|}{$\begin{array}{l}\text { ANOVA test followed by Bonferroni's post- } \\
\text { test } v s \text { control group }(\mathrm{p})\end{array}$} & ns & ns & ns & ns & ns & ns \\
\hline \multirow{7}{*}{ 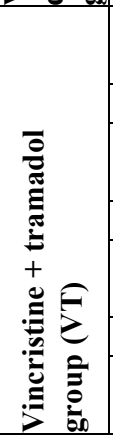 } & $\begin{array}{l}\text { Mean/group } \quad \pm \\
\text { SEM }\end{array}$ & \begin{tabular}{ll|}
8.55 & \pm \\
0.94 & \\
\end{tabular} & $\begin{array}{ll}33.29 \quad \pm \\
2.64\end{array}$ & $\begin{array}{ll}6.67 & \pm \\
1.63 & \\
\end{array}$ & $\begin{array}{ll}27.33 \quad \pm \\
6.54\end{array}$ & $\begin{array}{ll}8.70 & \pm \\
1.17 & \\
\end{array}$ & $\begin{array}{ll}33.41 \pm \\
3.35\end{array}$ & $\begin{array}{ll}9.16 \quad \pm \\
2.35\end{array}$ & $\begin{array}{ll}34.12 \pm \\
3.74\end{array}$ \\
\hline & \multicolumn{3}{|c|}{ Procentual change $v s$ baseline } & -21.96 & -17.90 & 1.76 & 0.36 & 7.15 & 2.49 \\
\hline & \multicolumn{3}{|c|}{$\begin{array}{l}\text { ANOVA test followed by Dunnett's post-test } \\
\text { vs baseline (p) }\end{array}$} & $*$ & $* *$ & ns & ns & ns & ns \\
\hline & \multicolumn{3}{|c|}{ Procentual change vs control group } & -27.77 & -19.00 & 1.64 & 0.64 & 3.16 & -2.19 \\
\hline & \multicolumn{3}{|c|}{$\begin{array}{l}\text { ANOVA test followed by Bonferroni's post- } \\
\text { test } v s \text { control group }(\mathrm{p})\end{array}$} & $*$ & $* *$ & ns & ns & ns & ns \\
\hline & \multicolumn{3}{|c|}{ Procentual change vs vincristine group } & 0.23 & 3.34 & 19.12 & 16.12 & 4.8 & -3.34 \\
\hline & \multicolumn{3}{|c|}{$\begin{array}{l}\text { ANOVA test followed by Bonferroni's post- } \\
\text { test vs paclitaxel group (p) }\end{array}$} & ns & ns & $* *$ & $* * *$ & $\mathrm{~ns}$ & ns \\
\hline
\end{tabular}

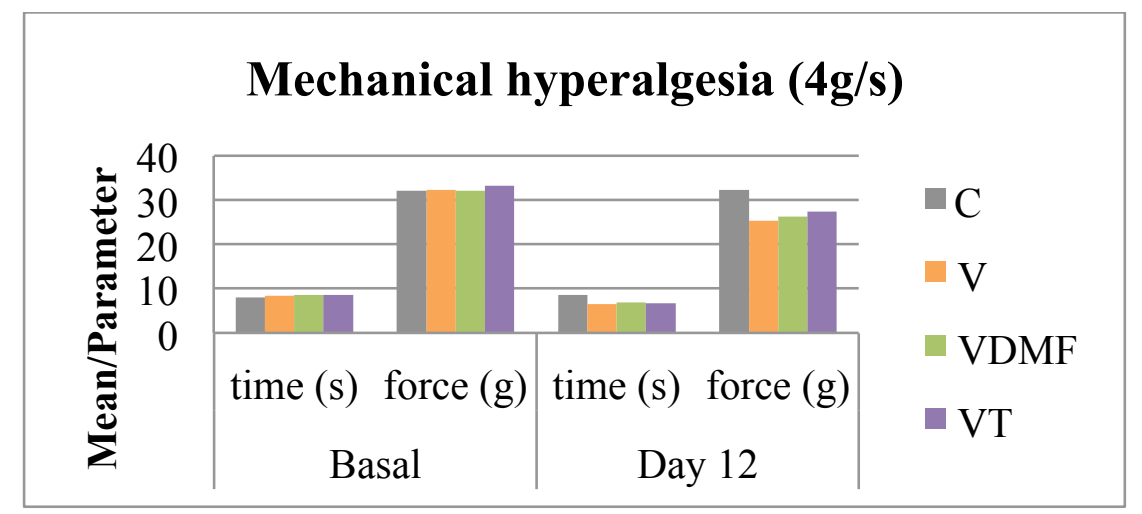

Figure 3.

Behavioural mechanical hyperalgesia induced with vincristine, shown as the mean of the time to response and applied force, to mechanical stimulation $4 \mathrm{~g} / \mathrm{s}$ 


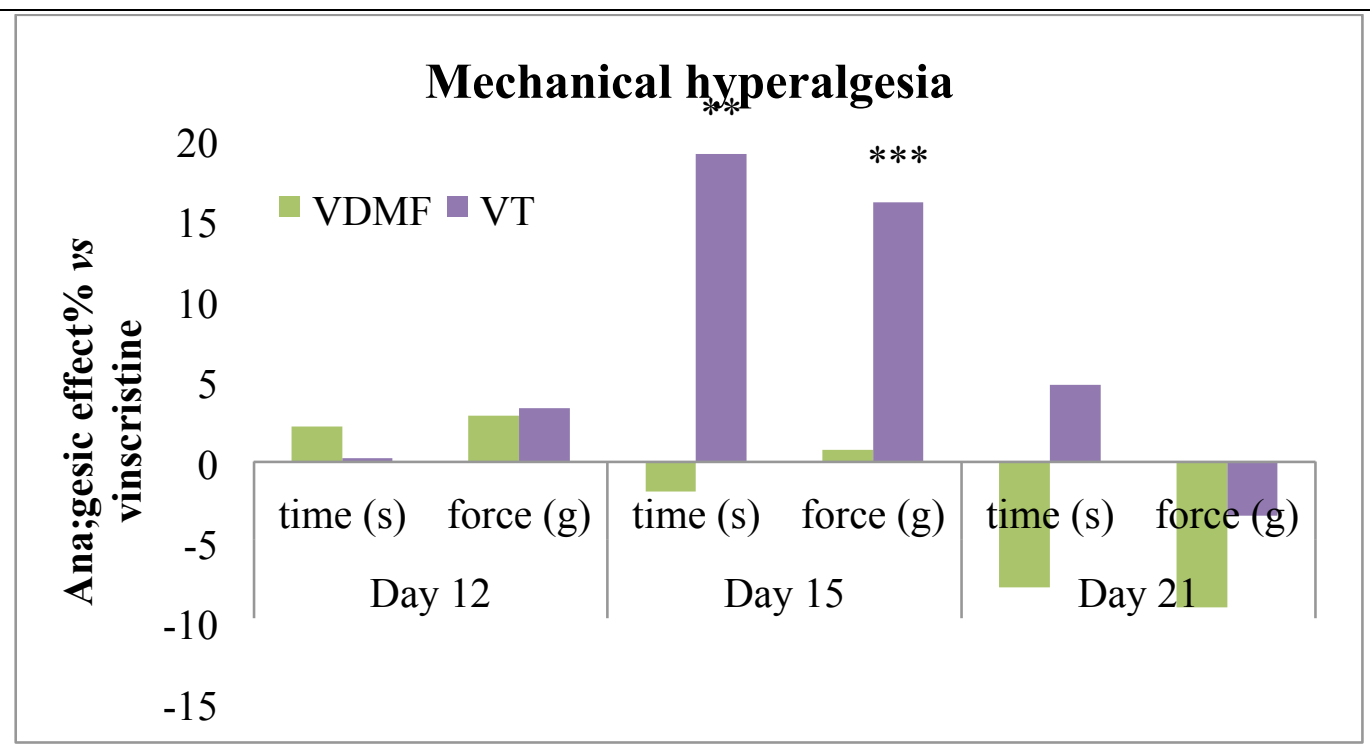

Figure 4.

Analgesic effect (\%) of dextromethorphan $(20 \mathrm{mg} / \mathrm{kg} \mathrm{bw})$ and tramadol (5 mg/kg bw) in the mechanical hyperalgesia induced with vincristine. Group comparisons were performed using the One-Way analysis of variance ANOVA followed by Bonferroni's test in comparison to vincristine group $* * \mathrm{p}<0.01, * * * \mathrm{p}<0.001$

Up to $80 \%$ of patients receiving chemotherapy experience chemotherapy-induced peripheral neuropathic pain (CIPNP) which is a severe adverse effect that can limit dose and choice of chemotherapy. Mechanisms involved in CIPNP include disruption of axonal transport, altered ion channel and receptor activity, neuronal injury and inflammation, oxidative stress, and mitochondrial damage [17]. The most frequent substances causing CIPNP are platin compounds, Vinca alkaloids, taxanes and bortezomib. The peripheral neuropathy of vincristine is severe, persist throughout the course of treatment and most often reversible after cessation [18]. The purpose of our research was to study the effect of tramadol and dextromethorphan on the peripheral neuropathy induced by vincristine in rats. We chose to investigate the action of two opioid analgesics (tramadol and dextromethorphan) recommended by the NeuPSIG 2015 guidelines in as second line of neuropathic pain treatment [12].

Allodynia and hyperalgesia following the administration of vincristine are highlighted by reducing the time to response for paw retraction. The smallest tactile sensitivity threshold for the vincristine group is registered after 15 days, when the parameter time to response decreases $v s$ control group (allodynia -53.83\%, $\mathrm{p}<0.05$ ). The hypersensitivities to mechanical stimulation induce by vincristine was reversible. The mechanical sensitivity parameters returned to baseline after 9 days since discontinuation of vincristine (Table I).

The experimental results have shown the analgesic effect of tramadol and dextromethorphan, expressed especially by significantly increasing the time to response, in the assessment of allodynia
Remarkable effects in reducing allodynia were recorded for tramadol. Tramadol $5 \mathrm{mg} / \mathrm{kg}$ bw presented antiallodynic effect versus vincristine group, most intensively after a single administration $(141.73 \% ; p<0.001)$ (Table I, Figure 2).

The mechanical hyperalgesia induced by vincristine in rats was decreased only by tramadol. The highest analgesic effect was recorded after a single administration, when the time to response increased by $19.12 \%$ relative to the vincristine group ( $\mathrm{p}<$ 0.01) (Table II, Figure 4).

The efficacy of tramadol in reducing chemotherapy-induced neuropathy has been shown in other experimental studies $[14,15]$. These data are consistent with results reported in literature that show clinical efficacy of tramadol in the treatment of acute neuropathic pain [19].

\section{Conclusions}

In conclusion, our present data provide evidence that allodynia and hyperalgesia evoked by vincristine might be inhibited by dextromethorphan but utterly by tramadol.

The pharmacotherapy of neuropathic pain is open to clinical and experimental research of new potential drugs. Using animal models in experimental research provides us with important data for future investigations into the neurobiological underpinnings of chemotherapy-induced neuropathic pain during life.

\section{Conflict of interests}

The authors declare that they have no conflict of interests. 


\section{References}

1. Stone JB, DeAngelis LM, Cancer TreatmentInduced Neurotoxicity: A Focus on Newer Treatments. Nature reviews Clinical oncology, 2016; 13(2): 92-105.

2. Kannarkat G, Lasher EE, Schiff D, Neurologic complications of chemotherapy agents. Curr Opin Neurol., 2007; 20: 719-725.

3. Park SB, Krishnan AV, Lin CS, Goldstein D, Friedlander M, Kiernan MC, Mechanisms underlying chemotherapyinduced neurotoxicity and the potential for neuroprotective strategies. Curr Med Chem., 2008; 15: 3081-3094.

4. Quasthoff S, Hartung HP, Chemotherapy-induced peripheral neuropathy. J Neurol., 2002; 249: 9-17.

5. Wolf S, Barton D, Kottschade L, Grothey A, Loprinzi C, Chemotherapy-induced peripheral neuropathy:Prevention and treatment strategies. Eur J Cancer, 2008; 44: 1507-1515

6. Preda MA, Popa G, Karancsi OL, Musat O, Popescu SI, Munteanu M, Popa Z. Effectiveness of subconjunctival bevacizumab associated with a laser - based procedure in the treatment of neovascular glaucoma. Farmacia, 2018; 66 (4): 621-626.

7. Boyette-Davis JA, Cata JP, Driver LC, Novy DM, Bruel BM, Mooring DL, Wendelschafer-Crabb G, Kennedy WR, Dougherty PM, Persistent chemoneuropathy in patients receiving the plant alkaloids paclitaxel and vincristine. Cancer Chemother Pharmacol., 2013; 71(3): 619-626.

8. Siau C, Xiao W, Bennett GJ, Paclitaxel-and vincristine-evoked painful peripheral neuropathies: loss of epidermal innervation and activation of Langerhans cells. Exp Neurol., 2006; 201(2): 507514.

9. Cruccu G, Truini A, A review of neuropathic pain: From guidelines to clinical practice. Pain and Therapy, 2017; 6(1): 35-42.

10. Chiriță C, Cioroianu DM, Chiriță IC, Negreș S, Marian B, Zbârcea CE, Synthesis and pharmacological activity of new acyloximines derivatives. Farmacia, 2016; 64(1):61-66.
11. Ba X, Wang J, Zhou S, Luo, Peng Y, Yang S, Hao $Y$, Jin G, Cinobufacini protects against paclitaxelinduced peripheral neuropathic pain and suppresses TRPV1 up-regulation and spinal astrocyte activation in rats. Biomed Pharmacother., 2018; 12(108): 76-84.

12. Finnerup NB, Attal N, Haroutounian S, McNicol E, Baron R, Dworkin RH, Gilron I, Haanpää M, Hansson P, Jensen TS, Kamerman PR, Lund K, Moore A, Raja SN, Rice AS, Rowbotham M, Sena E, Siddall $\mathrm{P}$, Smith $\mathrm{BH}$, Wallace $\mathrm{M}$, Pharmacotherapy for neuropathic pain in adults: a systematic review and meta-analysis. Lancet Neurol., 2015; 14(2): 162-173.

13. Arbaiza D,Vidal O, Tramadol in the treatment of neuropathic cancer pain. Clin Drug Invest., 2007; 27(1): 75-83.

14. Zbârcea CE, Ciotu IC, Bild V, Chiriță C, Tănase $\mathrm{AM}$, Şeremet OC, Ştefănescu E, Arsene AL, Bastian AE, Ionică FE, Negreș S, Therapeutic potential of certain drug combinations on paclitaxel-induced peripheral neuropathy in rats. Rom J Morphol Embryol., 2017; 58(2): 507-516.

15. Zbârcea CE, Negreş S, Cristea AN, Chiriţă C. The effect of dextrometorphan, gabapentin, amitriptyline and tramadol on a mouse model of vincristine-induced peripheral neuropathy. Farmacia, 2011; 59(6): 809-819.

16. Weng HR, Cordella JV, Dougherty PM, Changes in sensory processing in the spinal dorsal horn accompany vincristine-induced hyperalgesia and allodynia. Pain., 2003; 103: 131-138.

17. Sisignano M, Baron R, Scholich K, Geisslinger G, Mechanism-based treatment for chemotherapyinduced peripheral neuropathic pain. Nat Rev Neurol., 2014; 10(12): 694-707.

18. Grisold W, Cavaletti G, Windebank AJ, Peripheral neuropathies from chemotherapeutics and targeted agents: diagnosis, treatment, and prevention. Neuro-Oncology., 2012; 14(Suppl 4): iv45-iv54.

19. Eisenberg E, McNicol ED, Carr DB, Efficacy of muopioid agonists in the treatment of evoked neuropathic pain: systematic review of randomized controlled trials. Eur J Pain., 2006; 10: 667-676. 